\title{
Blockade of corticotropin-releasing factor receptor 1 in the central amygdala prevents cocaine-seeking behaviour induced by orexin-A administered to the posterior paraventricular nucleus of the thalamus in male rats
}

\author{
Alessandra Matzeu, PhD; Rémi Martin-Fardon, PhD
}

\begin{abstract}
Background: Orexin-A (OrxA) administration in the posterior paraventricular nucleus of the thalamus ( $p P V T)$ reinstates extinguished cocaine-seeking behaviour following extended access to the drug (a model of dependence). The pPVT receives and integrates information associated with emotionally salient events and sends excitatory inputs to brain regions involved in the expression of emotional states, such as those driving cocaine-seeking behaviour (i.e., the nucleus accumbens, the central nucleus of the amygdala [CeA], the basolateral amygdala, the bed nucleus of the stria terminalis [BNST] and the prefrontal cortex). Methods: We monitored the activation pattern of these regions (measured by Fos) during cocaine-seeking induced by OrxA administered to the pPVT. The BNST and CeA emerged as being selectively activated. To test whether the functionality of these regions was pivotal during OrxA-induced cocaine-seeking behaviour, we transiently inactivated these regions concomitantly with OrxA administration to the pPVT. We then tested the participation of corticotropin-releasing factor receptors $\left(\mathrm{CRF}_{1}\right)$ in the CeA during OrxA-induced cocaine-seeking using the CRF $F_{1}$ antagonist $\mathrm{CP}_{154526}$. Results: We observed selective activation of the CeA and BNST during cocaine-seeking induced by OrxA administered to the pPVT, but only transient inactivation of the CeA prevented cocaine-seeking behaviour. Administration of CP154526 to the CeA prevented OrxAinduced cocaine-seeking behaviour. Limitations: The use of only male rats could have been a limitation. Other limitations could have been the use of an indirect approach to test the hypothesis that administration of OrxA to the pPVT drives cocaine-seeking via CRF $_{1}$ signalling in the $\mathrm{CeA}$, and a lack of analysis of the participation of $\mathrm{CeA}$ subregions. Conclusion: Cocaine-seeking behaviour induced by OrxA administered to the pPVT is driven by activation of the $\mathrm{CeA}$ via $\mathrm{CRF}_{1}$ signalling.
\end{abstract}

\section{Introduction}

The paraventricular nucleus of the thalamus (PVT) has gained considerable attention in the study of drug-related behaviours because of its prominent projections to pivotal components of the neurocircuitry of addiction. ${ }^{1}$ The PVT is strongly activated by a wide range of stressful and arousing stimuli (e.g., Hamlin and colleagues, ${ }^{2}$ James and colleagues ${ }^{3}$ and Bubser and Deutch ${ }^{4}$ ) and is selectively recruited during cocaine-seeking behaviour. ${ }^{5}$ The integrity of the PVT is necessary for behaviour that is motivated by the presentation of cocaine-associated environmental stimuli. ${ }^{6}$ The PVT might act as a hub that receives and integrates emotionally salient events, which in turn are transmitted to other brain regions that regulate diverse behavioural outcomes.

The PVT receives dense peptidergic inputs from the hypothalamus. ${ }^{7,8}$ The posterior PVT (pPVT) receives some of the densest orexin projections in the brain. ${ }^{7,9}$ The orexin (or hypocretin) system regulates physiologic functions ${ }^{10,11}$ and modulates stress ${ }^{12}$ and reward states (e.g., drug-seeking behaviour). ${ }^{13-15}$ Modulatory functions of the orexin system in arousal, stress and reward are partially mediated by the depolarization of PVT neurons. ${ }^{16}$ Importantly, orexin transmission in the pPVT has been implicated in reward-seeking behaviour. ${ }^{17,18}$ Microinjections of orexin-A (OrxA) in the pPVT reinstated (primed) both cocaine- and food-seeking behaviour, with a greater effect in animals trained to self-administer cocaine. ${ }^{17,18}$

The PVT receives and integrates information associated with emotionally salient events and then sends excitatory inputs to brain regions involved in the expression of emotional states, such as those that drive cocaine-seeking behaviour (i.e., the nucleus accumbens, the central nucleus of the amygdala [CeA], the basolateral amygdala, the bed nucleus of the stria terminalis [BNST] and the prefrontal cortex). ${ }^{19}$ In the present study, we

Correspondence to: A. Matzeu, Department of Molecular Medicine, the Scripps Research Institute, 10550 North Torrey Pines Road, SR-107, La Jolla, CA 92037, USA; amatzeu @ scripps.edu

Submitted Nov. 18, 2020; Revised Jan. 25, 2021; Accepted Mar. 11, 2021

DOI: $10.1503 / j p n .200213$ 
determined activation patterns (i.e., recruitment) of these regions (measured by Fos expression) during cocaine-seeking behaviour induced by OrxA administered to the pPVT. We sought to determine whether any of these brain regions were activated exclusively during cocaine-seeking behaviour, and whether they were pivotal for the expression of cocaine-seeking behaviour induced by OrxA. We used Fos mapping to determine the neural substrates through which intra-pPVT administration of OrxA exerts a priming effect to induce the reinstatement of extinguished cocaine-seeking behaviour in a rat model of cocaine dependence (i.e., cocaine long access [C-LA]). The BNST and $\mathrm{CeA}$ emerged as the only brain regions that were selectively activated during cocaine-seeking induced by OrxA administered to the pPVT in C-LA animals. However, only CeA functionality was necessary for the reinstatement of cocaine-seeking behaviour. The corticotropin-releasing factor (CRF) system is strongly engaged in negative emotional states that characterize drug withdrawal during abstinence..$^{20,21}$ Thus, we injected a corticotropin-releasing factor receptor $1\left(\mathrm{CRF}_{1}\right)$ antagonist in the $\mathrm{CeA}$ to test whether $\mathrm{CRF}_{1}$ receptors in the CeA played a role in cocaine-seeking induced by OrxA administered to the pPVT.

\section{Methods}

\section{Animals}

Male Wistar rats ( $n=118$, Charles River), weighing 200 to $225 \mathrm{~g}$ upon arrival, were housed 2 per cage in a temperature- and humidity-controlled vivarium on a reverse light/dark cycle (12 h/12 h) with ad libitum access to food and water. All procedures were conducted during the dark phase of the light/ dark cycle and with strict adherence to the National Institutes of Health Guide for the Care and Use of Laboratory Animals. ${ }^{22}$ The animal procedures were approved by the Institutional Animal Care and Use Committee of The Scripps Research Institute.

\section{Drugs}

Orexin-A (American Peptide) was diluted in $0.9 \%$ sodium chloride (Hospira) and injected in volumes of $0.5 \mu \mathrm{L}$. Cocaine (National Institute on Drug Abuse) was dissolved in $0.9 \%$ sodium chloride and administered at a dose of $0.25 \mathrm{mg} / 0.1 \mathrm{~mL}$. Sweetened condensed milk (Nestlé) was diluted 2:1 (vol./ vol.) in water and delivered in volumes of $0.1 \mathrm{~mL}$. Muscimol and baclofen (Tocris Bioscience) were dissolved in $0.9 \%$ sodium chloride at concentrations of 0.6 and $0.06 \mathrm{mM}$, respectively. Finally, CP154526 (Tocris Bioscience) was dissolved in 100\% dimethylsulfoxide (Sigma Aldrich).

\section{Experimental procedures}

We prepared 4 separate experimental groups of rats. Detailed methods for each experimental group can be found in Appendix 1, available at jpn.ca/200213-a1.

\section{Group 1: Reinstatement of cocaine-seeking behaviour:} brain activation patterns in C-LA rats

Rats that were designated for C-LA self-administration were trained to self-administer cocaine in daily 6-hour sessions. Fourteen days after they began self-administration training, the rats were stereotaxically implanted with a guide cannula aimed at the pPVT. After recovery (7 days), they resumed self-administration training for 7 more days.

Immediately after 21 daily self-administration sessions, the rats underwent extinction training. On the last day of extinction training, each rat received a sham injection for habituation to the microinjection. Then, 24 hours later, they received an intra-pPVT microinjection of $0.5 \mu \mathrm{g}$ OrxA or vehicle. Half of the rats were then placed in the operant chambers under extinction conditions for 2 hours and tested for the reinstatement of cocaine-seeking behaviour. The other half remained in their home cage for 2 hours. This latter subgroup was used to control for possible effects of the operant chamber (in addition to the pPVT injection) on brain activation.

Immediately after the reinstatement session (or after the home cage), the rats were euthanized. Their brains were sectioned coronally and then processed for Fos immunodetection. Fos-positive (Fos+) neurons were counted in sections that incorporated the pPVT, BNST, CeA, prelimbic cortex, infralimbic cortex, nucleus accumbens core, nucleus accumbens shell and basolateral amygdala. Injection tracks were verified, and off-target cannulations were excluded from the study. For immunohistochemistry, we prepared 1 agematched group of naive rats $(n=6)$.

\section{Group 2: Reinstatement of cocaine-seeking behaviour: inactivation of the $\mathrm{BNST}$ and CeA in C-LA rats} In the experiments for group 1, the BNST and CeA emerged as being selectively activated during the intrapPVT OrxA-induced reinstatement of cocaine-seeking behaviour in cocaine rats. To test whether the functionality of these regions was pivotal during cocaine-seeking behaviour, we combined transient inactivation of the BNST or CeA with intra-pPVT OrxA administration in another group of C-LA rats.

\section{Group 3: Reinstatement of reward-seeking behaviour and CeA activation pattern: short access to cocaine versus sweetened condensed milk}

In the experiments for group 2, we found that functionality of the CeA was necessary for the expression of cocaine-seeking behaviour in C-LA rats. To exclude the possibility that this finding reflected general activation that occurred during cocaine-seeking behaviour induced by OrxA administered to the pPVT, we analyzed CeA activation patterns in rats after cocaine short access (C-SA) or sweetened condensed milk self-administration.

\section{Group 4: Reinstatement of cocaine-seeking behaviour in C-LA rats: effect of $C_{R F_{1}}$ receptor blockade in the CeA} The CRF-CRF $F_{1}$ receptor system in the CeA plays a pivotal role in negative emotional states that characterize withdrawal during abstinence. This experiment tested the effects of $\mathrm{CRF}_{1}$ receptor blockade in the CeA concomitant with OrxA injection in the pPVT in C-LA rats. 


\section{Statistical analysis}

We analyzed the acquisition of cocaine and sweetened condensed milk via self-administration using 2-way repeatedmeasures analysis of variance (ANOVA), with time (sessions) and lever (active $\mathrm{v}$. inactive) as factors. We analyzed reinstatement using 2-way ANOVA, with reinstatement condition (i.e., extinction, sham, vehicle, OrxA, muscimol + baclofen, and CP154526) and lever (active v. inactive) as factors. We analyzed histochemical data (i.e., the number of Fos+ neurons) using 1-way ANOVA for each brain region. Significant main effects or interactions were followed by the Sidak post hoc test. All results are expressed as mean \pm standard error of the mean (SEM). Values of $p<0.05$ were considered statistically significant. Statistical analyses were performed using Prism 8 software (GraphPad).

\section{Results}

Figure 1A shows the behavioural procedure used.

Seventeen rats were lost ( 6 because of health complications, 3 because of catheter failure and 8 because of cannula misplacement [7 for the pPVT and 1 for the BNST]), reducing the total number of rats to 101 (group 1, $n=29$, including 6 naive; group 2, $n=24$; group $3, n=30$, including 6 naive; group $4, n=18)$.
Group 1: Reinstatement of cocaine-seeking behaviour: brain activation patterns in $C-L A$ rats

\section{Self-administration and extinction}

Over the 21 days of self-administration training $(6 \mathrm{~h} / \mathrm{d})$, the rats $(n=23)$ acquired cocaine self-administration (2-way ANOVA: session $F_{20,880}=33.67, p<0.001$; lever $F_{1,44}=$ $311.44, p<0.001$; session $\times$ lever interaction $F_{20,880}=54.11$, $p<0.001$; Figure 1B). The Sidak post hoc test confirmed that the rats increased their cocaine intake in session 3 compared to session $1(p<0.05)$. Starting in session 4 , they emitted more responses on the active lever than on the inactive lever $(p<0.01)$. At the end of extinction training $(16 \pm 2$ sessions), the rats reached a mean \pm SEM of $6.17 \pm$ 0.76 responses on the active lever.

Reinstatement induced by OrxA administered to the pPVT Following extinction, the rats received a sham injection in the pPVT to habituate them to the injection procedure. The injection of OrxA in the pPVT reinstated (primed) cocaineseeking behaviour compared to extinction and the sham and vehicle injections $(p<0.001$; Sidak post hoc test following 2-way ANOVA: treatment $F_{3,58}=14.55, p<0.001$; lever $F_{1,58}=21.66, p<0.001$; treatment $\times$ lever interaction $F_{3,58}=$ $10.01, p<0.001$; Figure 1C) without altering responses on the inactive lever.

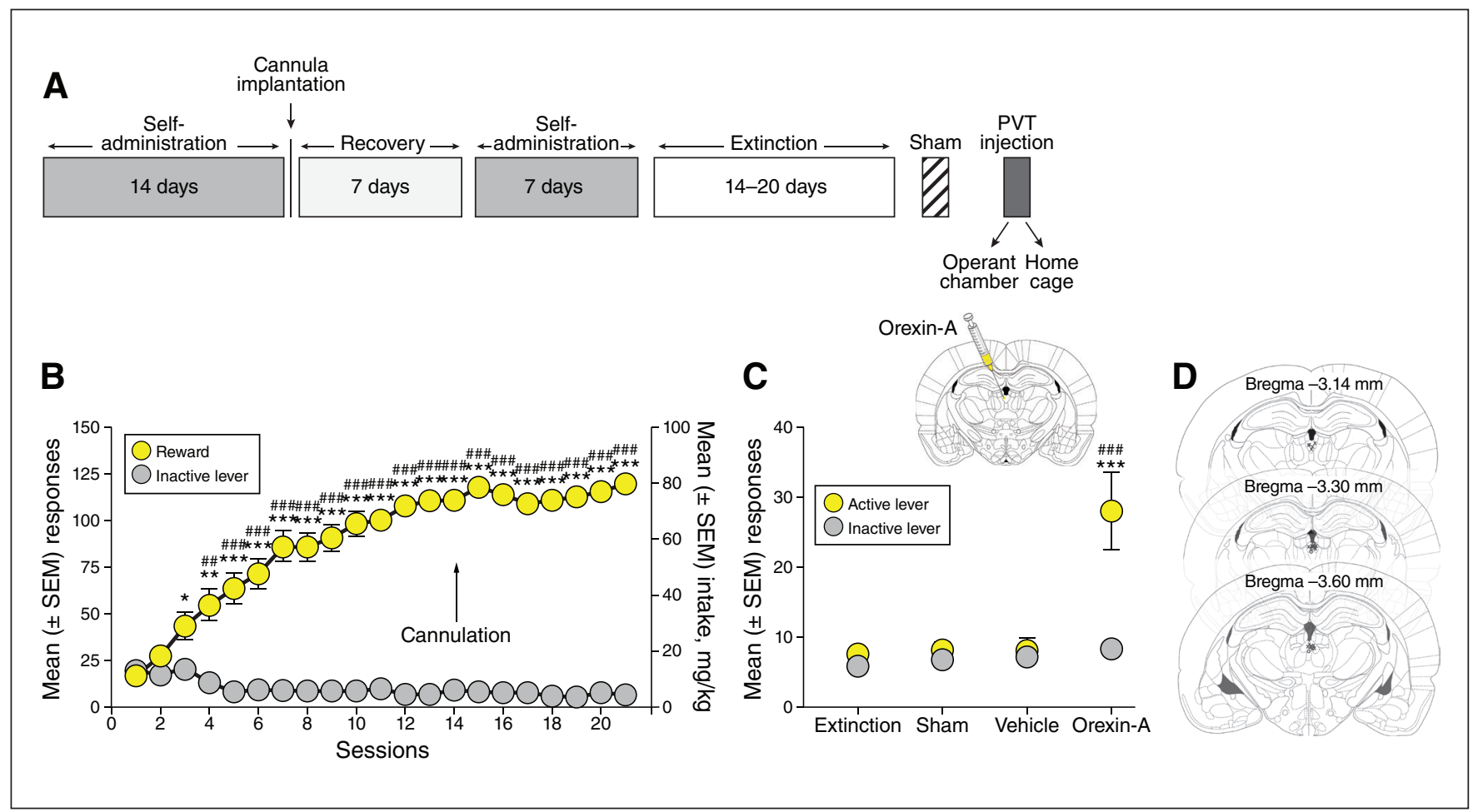

Figure 1: (A) Behavioural procedure. (B) Group 1. Time course of cocaine long access self-administration over 21 days of training (Sidak post

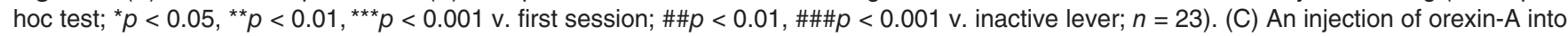
the posterior paraventricular nucleus of the thalamus induced cocaine-seeking behaviour (Sidak post hoc test; ${ }^{* \star *} p<0.001 \mathrm{v}$. extinction, sham and vehicle; \#\#\#p < $0.001 \mathrm{v}$. inactive lever; $n=5-11$. (D) Schematic distribution of injection site tracks $(\mathrm{x}=$ rats with correct cannula placements; $\mathrm{O}=$ rats with incorrect cannula placements). PVT = paraventricular nucleus of the thalamus; SEM = standard error of the mean. 


\section{Fos immunoreactivity}

Fos expression increased in the pPVT (Figure 2A to G) following vehicle or OrxA injection, but we observed the densest Fos expression in the pPVT following OrxA injection when the rats were placed in the operant chambers and allowed to press levers (1-way ANOVA: $F_{4,24}=28.24$, $p<0.001$; Sidak post hoc test: $p<0.05 \mathrm{v}$. orexin operant chamber; Figure 2G).

The number of Fos+ neurons in the BNST (Figure $2 \mathrm{H}$ to $\mathrm{N}$ ) was significantly higher following OrxA injection when the rats were placed in the operant chambers and tested for cocaine-seeking behaviour, without any significant activation
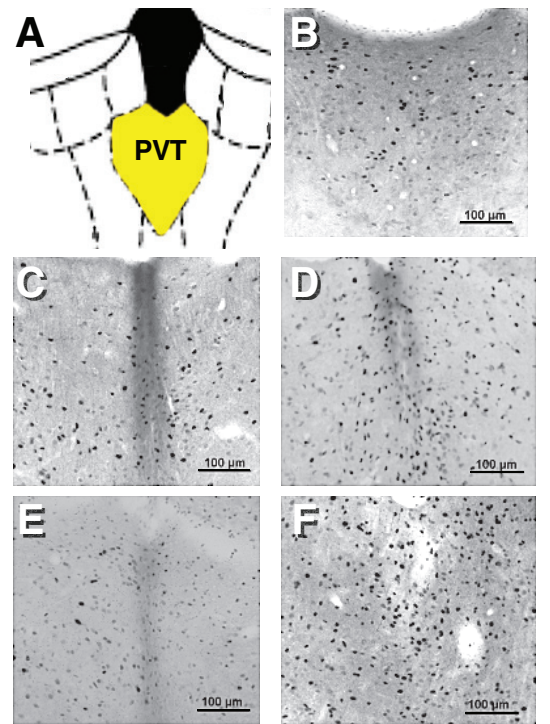

G

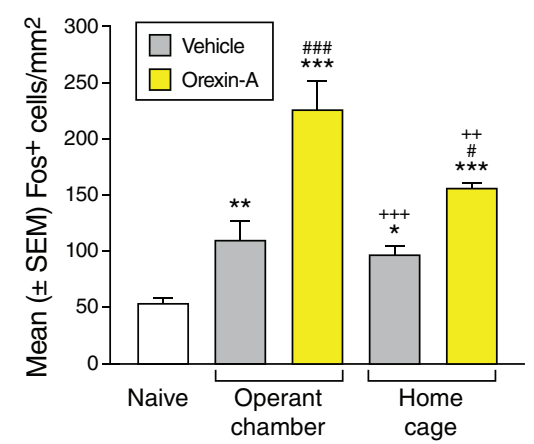

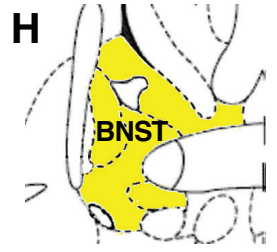
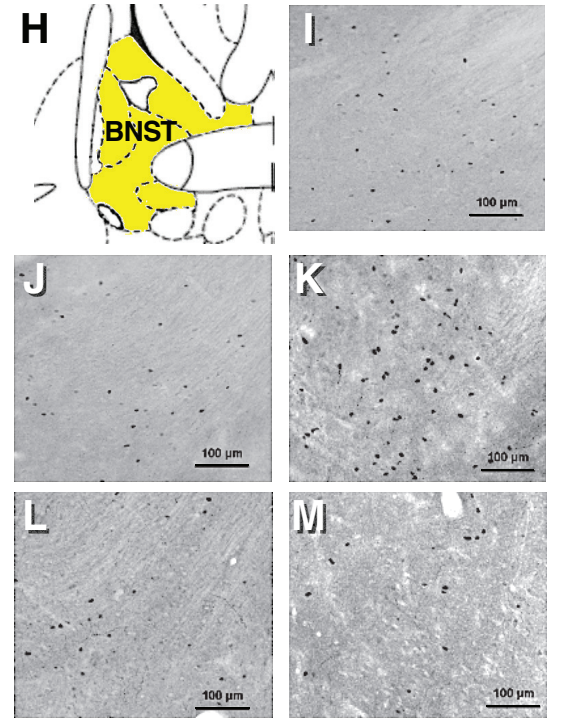

$\mathbf{N}$

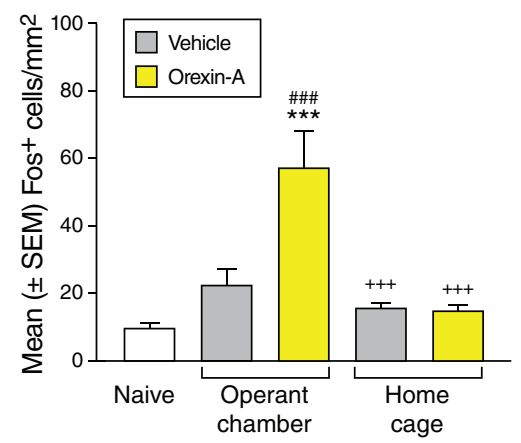

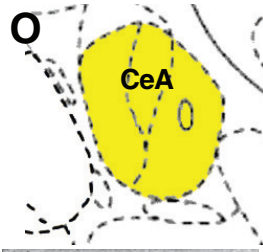
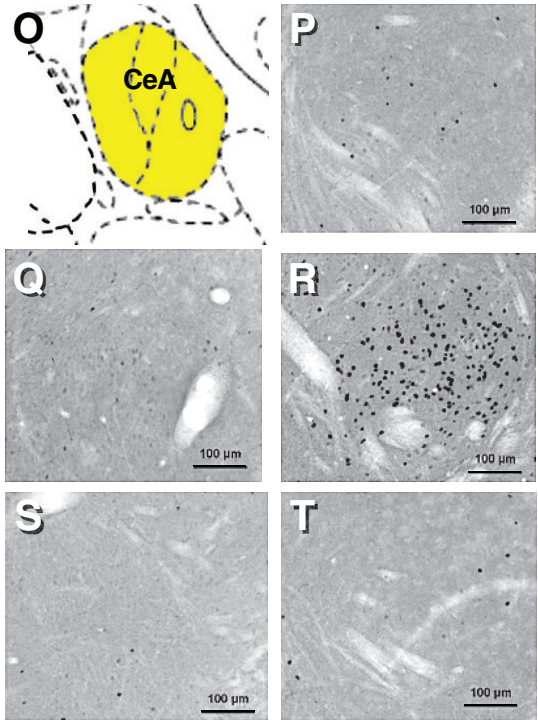

$\mathbf{U}$

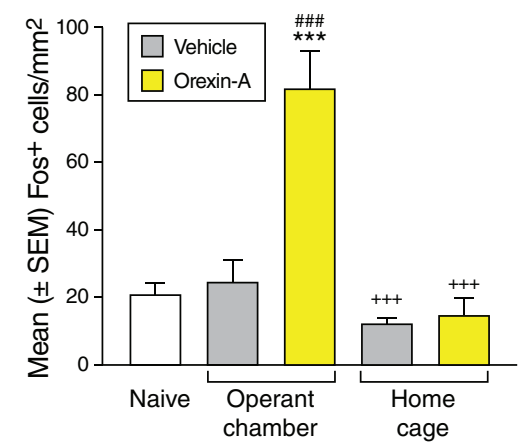

Figure 2: Activation patterns in the pPVT, BNST and CeA following an intra-pPVT injection of orexin-A to induce the reinstatement of cocaineseeking behaviour; $n=5-6$ animals/group. (A) Schematic illustration of the rostrocaudal level of cannula placement where pPVT Fos+ neurons were counted. Typical photomicrographs illustrate Fos activation and injection tracks in the pPVT in (B) naive rats; rats that were injected with $(C)$ vehicle or (D) orexin-A and exposed to the operant chamber; and rats that were injected with (E) vehicle or $(F)$ orexin-A and exposed to the home cage. (G) Number of Fos+ neurons in the pPVT following vehicle or orexin-A injection (Sidak post hoc test; ${ }^{*} p<0.05$, ${ }^{* *} p<0.01$,

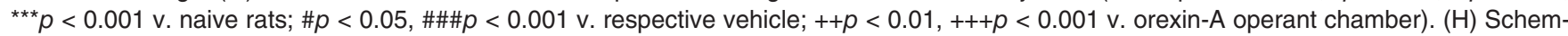
atic illustration of the rostrocaudal level where BNST Fos+ neurons were counted. Typical photomicrographs illustrate Fos activation in the BNST in (I) naive rats; rats that were injected with $(\mathrm{J})$ vehicle or $(\mathrm{K})$ orexin-A and exposed to the operant chamber; and rats that were injected with (L) vehicle or (M) orexin-A and exposed to the home cage. (N) Number of Fos+ neurons in the BNST following intra-pPVT orexin-A injec-

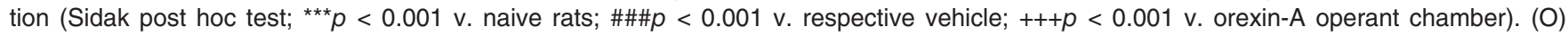
Schematic illustration of the rostrocaudal level where CeA Fos+ neurons were counted. Typical photomicrographs illustrate Fos activation in the $\mathrm{CeA}$ in $(\mathrm{P})$ naive rats; rats that were injected with $(\mathrm{Q})$ vehicle or $(\mathrm{R})$ orexin-A and exposed to the operant chamber; and rats that were injected with (S) vehicle or (T) orexin-A and exposed to the home cage. (U) Number of Fos+ neurons in the CeA following intra-pPVT orexin-A injection (Sidak post hoc test; ${ }^{* *} p<0.001 \mathrm{v}$. naive rats; \#\#\#p $<0.001 \mathrm{v}$. vehicle; $+++p<0.001 \mathrm{v}$. orexin-A operant chamber). BNST = bed nucleus of the stria terminalis; $\mathrm{CeA}=$ central nucleus of the amygdala; $\mathrm{pPVT}=$ posterior paraventricular nucleus of the thalamus; PVT = paraventricular nucleus of the thalamus; $\mathrm{SEM}=$ standard error of the mean. 
in any other condition (1-way ANOVA: $F_{4,24}=15.82, p<0.001$; Sidak post hoc test: $p<0.001 \mathrm{v}$. all other conditions; Figure $2 \mathrm{~N})$.

We also observed an increase in Fos expression in CeA neurons (Figure $2 \mathrm{O}$ to $\mathrm{U}$ ) when the rats were placed in the operant chambers and tested for cocaine-seeking behaviour following OrxA injection (1-way ANOVA: $F_{4,24}=22.09, p<$ 0.001; Sidak post hoc test: $p<0.001, \mathrm{v}$. all other conditions; Figure 2U). We observed no increase in Fos+ neurons in any of the other conditions. In parallel with the pPVT, BNST and CeA, we also counted Fos+ neurons in the prelimbic cortex, infralimbic cortex, nucleus accumbens core, nucleus accumbens shell and basolateral amygdala (Appendix 1, Figure S1).
Group 2: Reinstatement of cocaine-seeking behaviour: inactivation of the BNST and CeA in C-LA rats

\section{Self-administration and extinction}

Over the 21 days of self-administration training $(6 \mathrm{~h} / \mathrm{d})$, the rats $(n=24)$ acquired cocaine self-administration (2-way ANOVA: session $F_{20,920}=9.71, p<0.001$; lever $F_{1,46}=218.9, p<$ 0.001 ; session $\times$ lever interaction $F_{20,920}=14.13, p<0.001$; Figure $3 \mathrm{~A})$. The Sidak post hoc test confirmed that the rats increased their cocaine intake in session 4 compared to session $1(p<0.001)$, and they emitted more responses on the active lever in session 3 than on the inactive lever $(p<0.001)$. At the
A

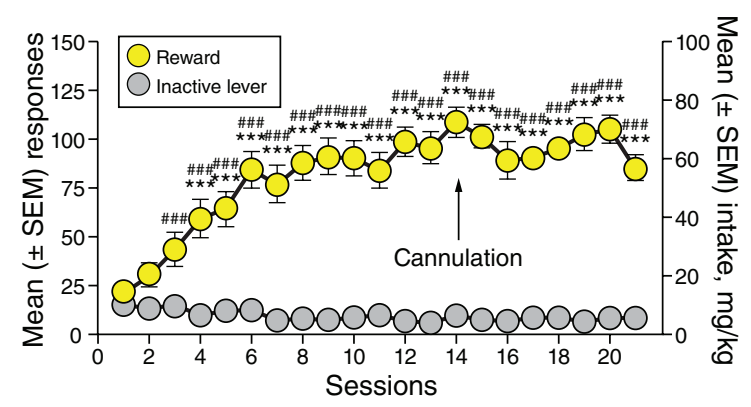

D

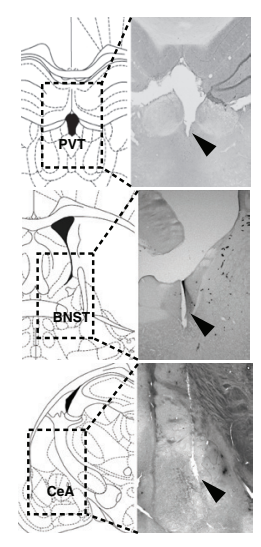

$\mathbf{E}$

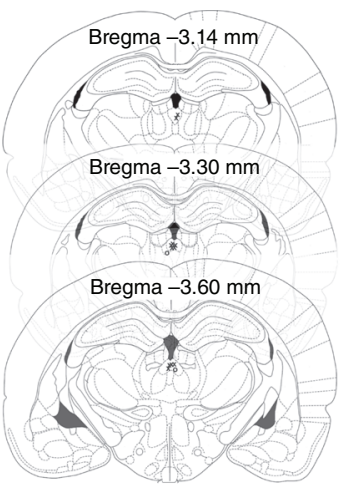

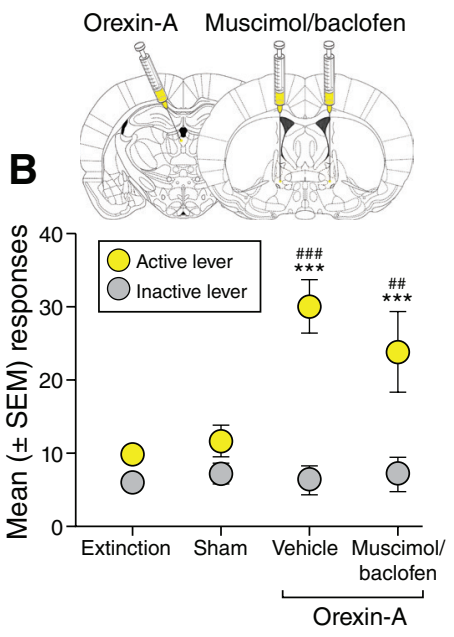
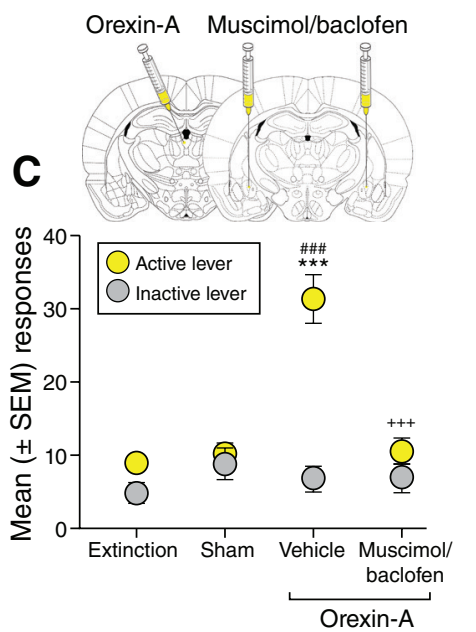

$\mathbf{F}$
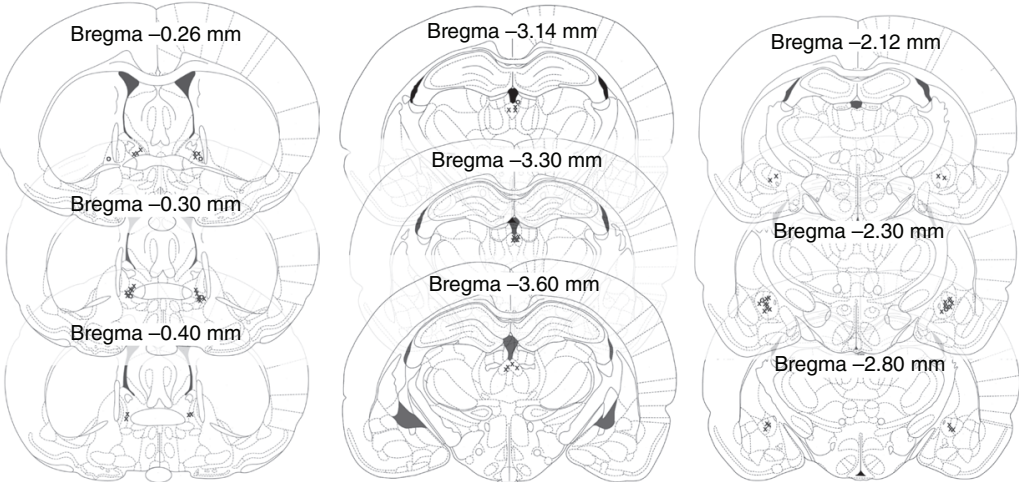

Figure 3: Transient inactivation of the CeA prevents cocaine-seeking behaviour induced by orexin-A administered to the pPVT. (A) Time

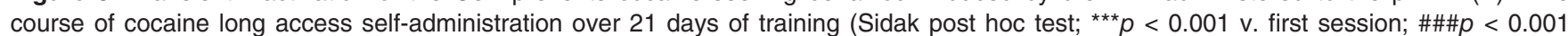
v. inactive lever; $n=24$ ). (B) Transient inactivation of the BNST did not affect the priming effect of intra-pPVT orexin-A on cocaine-seeking behaviour (Sidak post hoc test; ${ }^{* *} p<0.001 \mathrm{v}$. extinction and sham; \#\#\#p $<0.001 \mathrm{v}$. inactive lever; $n=5-11$ ). (C) Transient inactivation of the $\mathrm{CeA}$ prevented the priming effect of intra-pPVT orexin-A on cocaine-seeking behaviour (Sidak post hoc test; ${ }^{* * *} p<0.001 \mathrm{~V}$. extinction and sham; \#\#\#p < $0.001 \mathrm{v}$. the inactive lever; $+++p<0.001 \mathrm{v}$. vehicle; $n=6-13)$. (D) Schematic representations of injector placements in the pPVT, BNST and CeA, with photo representations of the injection track (2x magnification). ( $E$ and $F)$ Schematic distribution of injection site tracks in the pPVT (E left, $F$ left), BNST (E right) and CeA ( $F$ right) subgroups ( $x=$ rats with correct cannula placements; $0=$ rats with incorrect cannula placements). BNST = bed nucleus of the stria terminalis; $\mathrm{CeA}=$ central nucleus of the amygdala; $\mathrm{pPVT}=$ posterior paraventricular nucleus of the thalamus; PVT = paraventricular nucleus of the thalamus; SEM = standard error of the mean. 
end of extinction training (17 \pm 1 sessions), the rats emitted a mean \pm SEM of $9.50 \pm 0.74$ responses on the active lever.

\section{Reinstatement induced by OrxA administered to the} pPVT and concomitant inactivation of the BNST or CeA Inactivation of the BNST did not influence the reinstatement of cocaine-seeking behaviour induced by intra-pPVT administration of OrxA (2-way ANOVA: treatment $F_{3,58}=8.48, p<$ 0.001 ; lever $F_{1,58}=54.14, p<0.001$; treatment $\times$ lever interaction $F_{3,58}=8.22, p<0.001$; Figure $3 \mathrm{~B}$ ), with no differences in the number of active lever presses between rats injected with muscimol + baclofen or vehicle.

In contrast, inactivation of the CeA completely prevented the reinstatement of cocaine-seeking behaviour induced by intra-pPVT administration of OrxA (2-way ANOVA: treatment $F_{3,70}=13.30, p<0.001$; lever $F_{1,70}=36.84, p<0.001$; treatment $\times$ lever interaction $F_{3,70}=12.78, p<0.001$; Sidak post hoc test: $p<0.001 \mathrm{v}$. vehicle; Figure 3C). Responses on the inactive lever were unaffected (Figure 3B and $C$ ).

\section{Group 3: Reinstatement of cocaine-seeking behaviour and CeA activation pattern: short access to cocaine and sweetened condensed milk}

\section{Cocaine short access}

Self-administration and extinction

Over the 21 days of self-administration training $(2 \mathrm{~h} / \mathrm{d})$, the rats $(n=12)$ acquired cocaine self-administration (2-way ANOVA: session $F_{20,440}=3.29, p<0.001$; lever $F_{1,22}=19.87$, $p<0.001$; session $\times$ lever interaction $F_{20,440}=9.12, p<0.001$; Figure $4 \mathrm{~A})$. The Sidak post hoc test confirmed that the rats increased their cocaine intake in session 13 compared to session $1(p<0.05)$, and they emitted more responses on the active lever starting in session 12 compared to the inactive

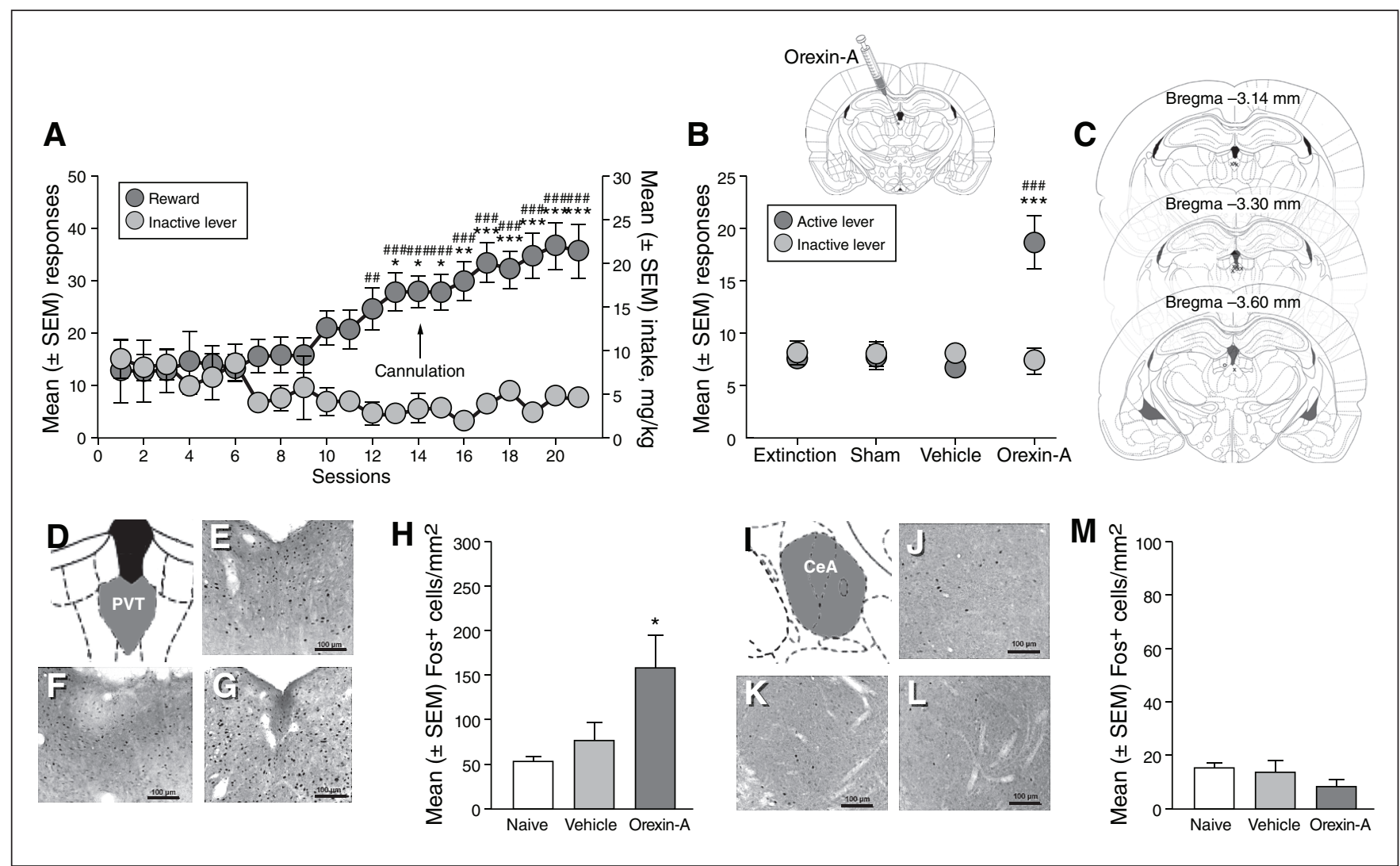

Figure 4: Activation pattern of the CeA following intra-pPVT injection of orexin-A to induce the reinstatement of cocaine-seeking behaviour in rats with short access to cocaine. (A) Time course of cocaine short access self-administration over 21 days of training (Sidak post hoc test;

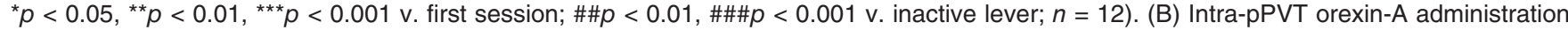
induced cocaine-seeking behaviour in rats with short access to cocaine (Sidak post hoc test; ${ }^{* * *} p<0.001 \mathrm{v}$. extinction, sham and vehicle; $\# \# \#<<0.001 \mathrm{v}$. inactive lever; $n=6-12)$. (C) Schematic distribution of injection site tracks $(\mathrm{x}=$ rats with correct cannula placements; $\mathrm{o}=$ rats with incorrect cannula placements). (D) Schematic illustration of the rostrocaudal level of cannula placement where pPVT Fos+ neurons were counted. Typical photomicrographs illustrate Fos activation and injection tracks in the pPVT in (E) naive rats and rats that were injected with $(F)$ vehicle or $(G)$ orexin-A. $(H)$ Number of Fos+ neurons in the pPVT following vehicle or orexin-A injections (Sidak post hoc test; ${ }^{*} p<0.05$ v. naive; $n=6$ /group). (I) Schematic illustration of the rostrocaudal level where CeA Fos+ neurons were counted. Typical photomicrographs illustrate Fos activation in $(\mathrm{J})$ naive rats and rats that were injected with (K) vehicle or (L) orexin-A. (M) Number of Fos+ neurons in the CeA ( $n=6$ /group). BNST $=$ bed nucleus of the stria terminalis; CeA $=$ central nucleus of the amygdala; pPVT = posterior paraventricular nucleus of the thalamus; PVT = paraventricular nucleus of the thalamus; SEM = standard error of the mean. 
lever $(p<0.01)$. At the end of extinction training $(15 \pm 1$ sessions), the rats emitted a mean \pm SEM of $7.6 \pm 1.0$ responses on the active lever.

Reinstatement induced by OrxA administered to the pPVT

The administration of OrxA to the pPVT reinstated cocaineseeking behaviour (2-way ANOVA: treatment $F_{3,64}=6.37, p<$ 0.001 ; lever $F_{1,64}=5.86, p<0.001$; treatment $\times$ lever interaction $F_{3,64}=8.33, p<0.001$; Sidak post hoc test: $p<0.001 \mathrm{v}$. extinction, sham and vehicle; Figure $4 \mathrm{~B}$ ). Responses on the inactive lever were unaffected (Figure 4B).

\section{Fos immunoreactivity}

Neurons in the pPVT (Figure 4D to G) were significantly activated (i.e., a higher number of Fos+ neurons) only after intra-pPVT OrxA injection compared to naive rats, but this increase was not different from vehicle injection (1-way
ANOVA: $F_{2,15}=5.630, p<0.05$; Sidak post hoc test: $p<0.05$ v. naive rats; Figure $4 \mathrm{H})$.

In the CeA (Figure 4I to L), Fos expression after OrxA or vehicle injection to the pPVT was unaffected and not different from that of naive rats (1-way ANOVA: $F_{2,15}=1.491$, $p=0.26$; Figure $4 \mathrm{M})$.

\section{Sweetened condensed milk}

Self-administration and extinction

The rats $(n=12)$ acquired sweetened condensed milk selfadministration in 30 minutes per session over 21 days (2way ANOVA: session $F_{20,440}=20.14, p<0.001$; lever $F_{1,22}=$ 632.7, $p<0.001$; session $\times$ lever interaction $F_{20,440}=30.51, p<$ 0.001; Figure 5A). Beginning in session 5 and compared to session 1, rats emitted more responses on the active versus inactive lever and maintained a high level of intake of sweetened condensed milk (Sidak post hoc test: $p<0.001$ ). At the
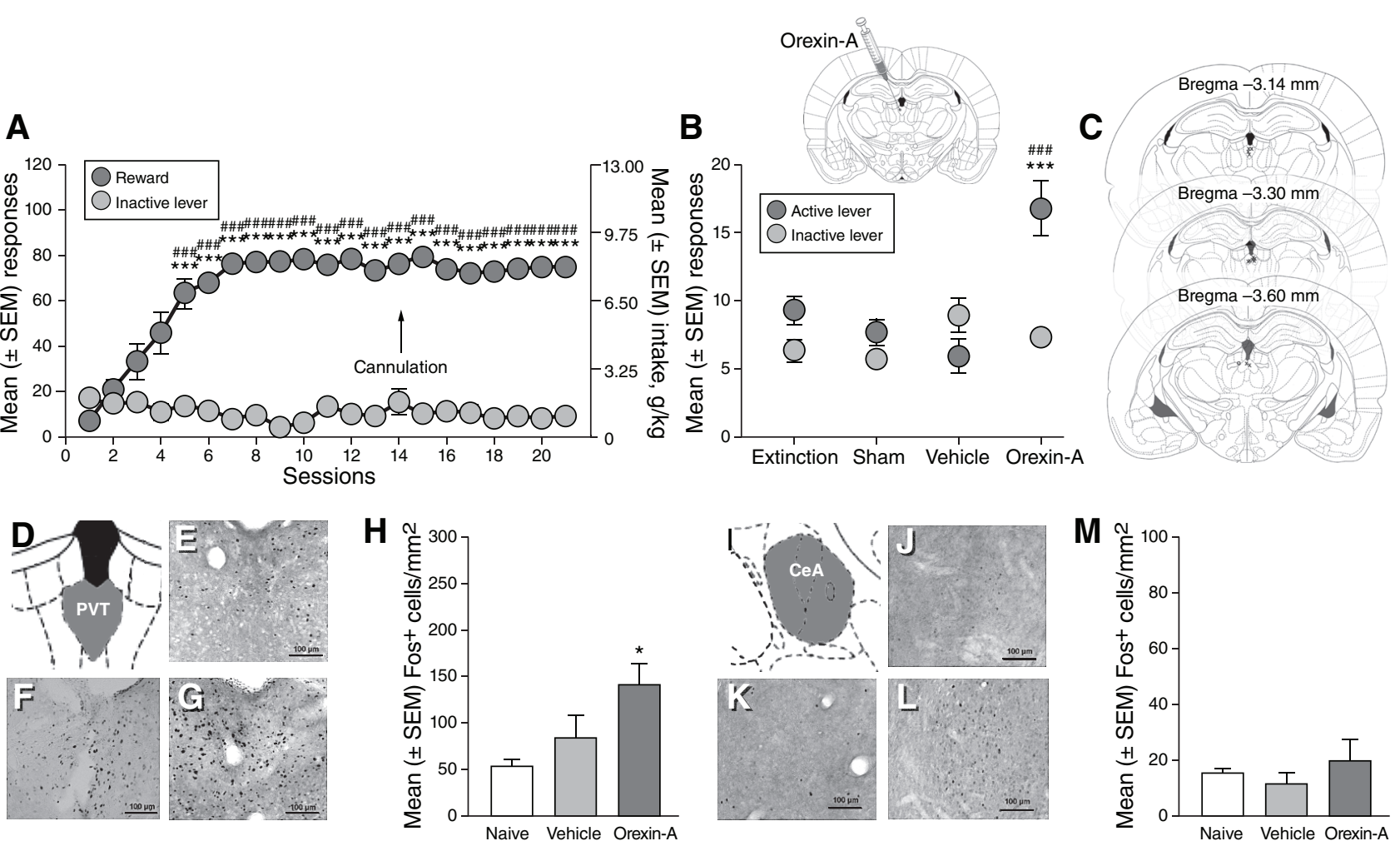

Figure 5: Activation pattern of the CeA following intra-pPVT injection of orexin-A to induce the reinstatement of sweetened condensed milkseeking behaviour. (A) Time course of sweetened condensed milk self-administration over 21 days of training (Sidak post hoc test; ${ }^{* * *} p<$ 0.001 v. first day; \#\#\#p < 0.001 v. inactive lever; $n=12$ ). (B) Intra-pPVT orexin-A administration primed sweetened condensed milk-seeking behaviour (Sidak post hoc test; ${ }^{* * *} p<0.001 \mathrm{v}$. extinction, sham and vehicle; \#\#\#p <0.001 v. inactive lever; $\left.n=6-12\right)$. (C) Schematic distribution of injection site tracks $(x=$ rats with correct cannula placements; $0=$ rats with incorrect cannula placements). (D) Schematic illustration of the rostrocaudal level of cannula placement where pPVT Fos+ neurons were counted. Typical photomicrographs illustrate Fos activation and injection track in the pPVT in (E) naive rats and rats that were injected with (F) vehicle or $(G)$ orexin-A. (H) Number of Fos+ neurons in the pPVT following vehicle or orexin-A injections (Sidak post hoc test; ${ }^{*} p<0.05$ v. naive; $n=6 /$ group). (I) Schematic illustration of the rostrocaudal level where CeA Fos+ neurons were counted. Typical photomicrographs illustrate Fos activation in $(\mathrm{J})$ naive rats and rats that were injected with $(\mathrm{K})$ vehicle or $(\mathrm{L})$ orexin-A. (M) Number of Fos+ neurons in the CeA ( $n=6 /$ group). BNST = bed nucleus of the stria terminalis; CeA = central nucleus of the amygdala; pPVT = posterior paraventricular nucleus of the thalamus; PVT = paraventricular nucleus of the thalamus; SEM = standard error of the mean. 
end of extinction training (18 \pm 2 sessions), the rats emitted a mean \pm SEM of $9.33 \pm 0.8$ responses for sweetened condensed milk.

\section{Reinstatement induced by OrxA administered to the $P P V T$}

The administration of OrxA to the pPVT produced significant reinstatement of sweetened condensed milk-seeking behaviour (2-way ANOVA: treatment $F_{3,64}=8.72, p<0.001$; lever $F_{1,64}=14.23, p<0.001$; treatment $\times$ lever interaction $F_{3,64}=$ 8.71, $p<0.001$; Sidak post hoc test: $p<0.001$, v. extinction, sham and vehicle; Figure 5B). Responses on the inactive lever remained low and unchanged (Figure 5B).

\section{Fos immunoreactivity}

We observed a significantly higher number of Fos+ neurons following the OrxA injection in the pPVT compared to naive rats (Figure $5 \mathrm{D}$ to $\mathrm{G}$ ), but this increase was not different from vehicle injection (1-way ANOVA: $F_{2,15}=4.584, p<0.05$; Sidak post hoc test: $p<0.05$, v. naive rats; Figure $5 \mathrm{H})$.

We observed no increase in the number of Fos+ neurons in the CeA (Figure 5I to L) after either vehicle or OrxA injection in the pPVT (1-way ANOVA: $F_{2,15}=0.7061, p=0.51$; Figure 5M).

\section{Group 4: Reinstatement of cocaine-seeking behaviour in $C-L A$ rats: effect of $C R F_{1}$ receptor blockade in the CeA}

\section{Self-administration and extinction}

Over the 21 days of self-administration training $(6 \mathrm{~h} / \mathrm{d})$, the rats $(n=18)$ acquired cocaine self-administration (2-way ANOVA: session $F_{20,680}=8.52, p<0.001$; lever $F_{1,34}=116.6, p<$ 0.001 ; session $\times$ lever interaction $F_{20,680}=17.1, p<0.001$; Figure 6A). The Sidak post hoc test confirmed that the rats emitted more responses on the active lever compared to the first session and compared to the inactive lever beginning in session $6(p<0.01)$. At the end of extinction training $(17 \pm$ 2 sessions), the rats emitted a mean \pm SEM of $8.77 \pm 0.89$ responses on the active lever.

\section{Reinstatement induced by OrxA administered to the} pPVT and concomitant $\mathrm{CRF}_{1}$ receptor blockade in the CeA The administration of CP154526 in the CeA prevented cocaineseeking behaviour induced by OrxA administered to the pPVT at the 2 doses tested, without affecting responses on the inactive lever (2-way ANOVA: treatment $F_{4,98}=13.46, p<0.001$; lever $F_{1,98}=57.16, p<0.001$; treatment $\times$ lever interaction $F_{4,98}=9.30$, $p<0.001$; Sidak post hoc test: $p<0.001$ v. vehicle; Figure 6B).

\section{Discussion}

The present study confirmed our previous observations that when injected in the pPVT, OrxA reinstates extinguished cocaine-seeking and sweetened condensed milk-seeking behaviour. ${ }^{17,18}$ Furthermore, the data indicate that the CeA is pivotal in cocaine-seeking behaviour induced by OrxA administered to the pPVT in rats with a history of C-LA (an animal model of cocaine dependence), but not in rats with C-SA or sweetened condensed milk exposure, and that $\mathrm{CRF}_{1}$ receptor signalling in the $\mathrm{CeA}$ mediates this behaviour. Overall, our results show that the connection between hypothalamic orexin inputs to the pPVT and the $\mathrm{CeA}$ is essential for inducing cocaine-seeking behaviour in cocaine-dependent rats with the participation of $\mathrm{CRF}_{1}$ receptors in the $\mathrm{CeA}$.

Before discussing the implications of these findings, a potentially important limitation of the present study needs to be discussed. A possible confound following the OrxA injection in the pPVT could have been that because of the close proximity of the pPVT to the third ventricle, the Fos activation we observed in the CeA and BNST could have resulted from OrxA-induced activation of nearby brain areas rather than the pPVT itself. However, the accuracy of the injections (depicted in Figures 1 to 6), together with our earlier studies that used a similar approach, $, 6,17,18,23$ strongly dispute this possibility. As reported previously, ${ }^{17,18}$ OrxA administration in the pPVT reinstated extinguished cocaine-seeking and sweetened condensed milk-seeking behaviour. Moreover, in C-LA rats, OrxA administration in the pPVT increased Fos expression, which was significantly higher when the animals were allowed to lever-press (i.e., during the reinstatement test), most likely because of the expression of cocaine-seeking behaviour. Studies that investigate the implications of endogenous orexin transmission in the pPVT during cocaine-seeking behaviour have been limited, and the mechanism by which OrxA administration in the pPVT triggers the reinstatement of reward-seeking behaviour is difficult to explain. The intraPVT administration of an orexin receptor 1 antagonist did not influence the cue-induced reinstatement of cocaine-seeking behaviour. ${ }^{24}$ The presentation of cues that were previously associated with ethanol availability activated PVT neurons that were in close contact with hypothalamic orexin-positive terminals. ${ }^{25}$ The administration of a dual orexin receptor antagonist in the pPVT prevented the stress-induced reinstatement of ethanol-seeking behaviour. ${ }^{23}$ These previous findings suggest that endogenous orexin transmission in the PVT (via orexin receptors) could play a pivotal role in drug-seeking behaviour. Additional studies are clearly required to define the role of endogenous orexin transmission in the PVT and implication of orexin receptor 1 versus orexin receptor 2 signalling during cue- and stress-induced cocaine-seeking behaviour. The present data strongly suggest selective participation of the CeA in reinstatement induced by OrxA administered to the PPVT in C-LA rats. One possibility could be that PPVT activation induced by OrxA generates anxiety and other aversive states that in turn elicit reinstatement. The PVT, especially the pPVT, receives extensive orexin inputs where orexin exerts strong excitatory effects. It has been suggested that the action of orexin in the pPVT is implicated in responses to stressful events. ${ }^{26}$ Long cocaine exposure alters the physiology of both orexin neurons and PVT neurons, ${ }^{27-29}$ and these changes could contribute to the "addiction" phenotype. The PVT receives information about emotionally charged events and in turn sends information to brain regions to regulate motivated behaviour. ${ }^{1}$ Indeed, the PVT is the only thalamic nucleus that projects to a group of structures that comprise the amygdala (i.e., $\mathrm{CeA}$ and basolateral amygdala), BNST, nucleus accumbens and prefrontal cortex..$^{30-32}$ 
A

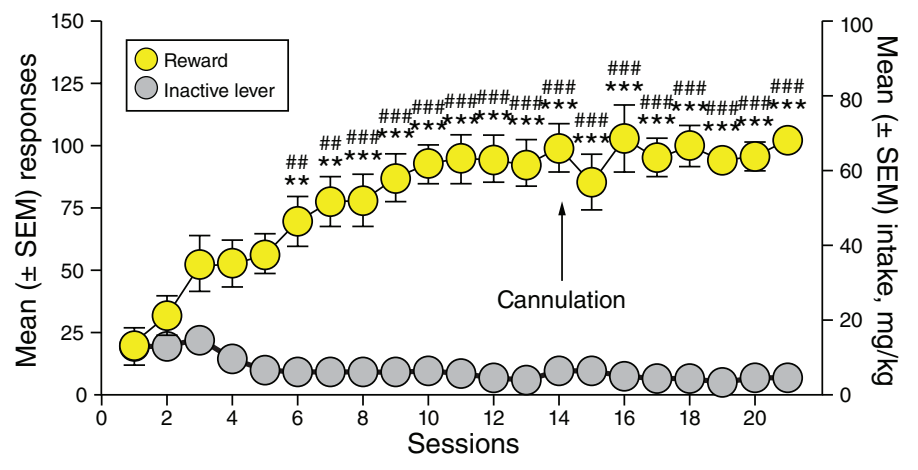

B
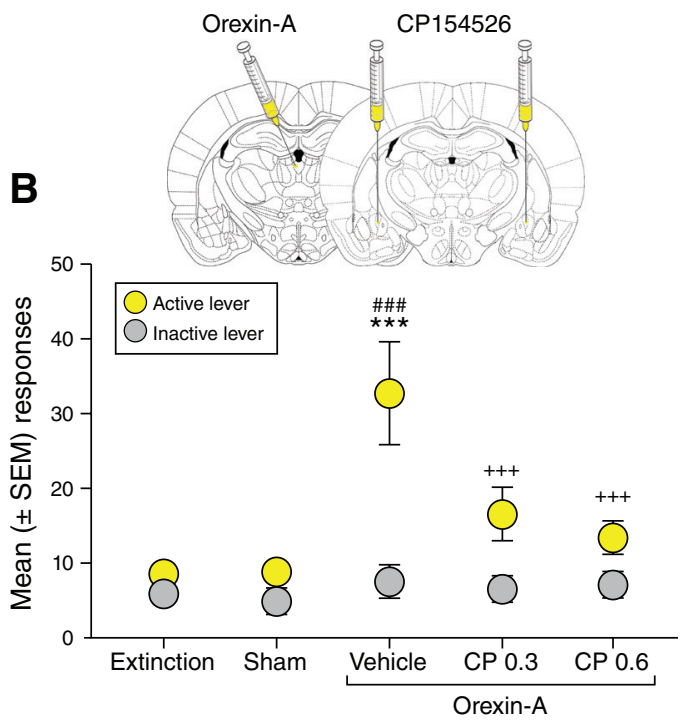

C
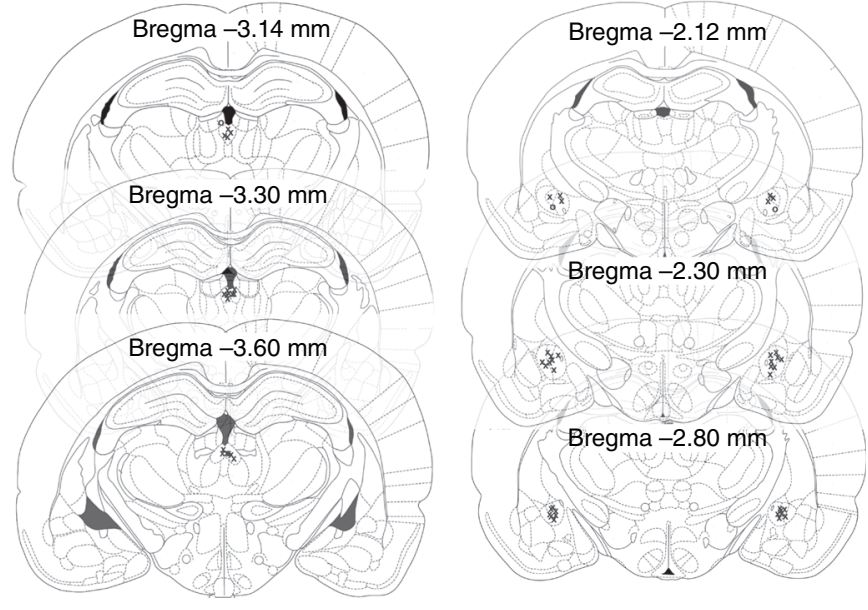

Figure 6: Blockade of $\mathrm{CRF}_{1}$ receptors in the CeA prevents cocaine-seeking behaviour induced by orexin-A administered to the pPVT. (A) Time course of cocaine long access self-administration over 21 days of training (Sidak post hoc test; ${ }^{* *} p<0.01,{ }^{* \star *} p<0.001 \mathrm{v}$. first day; $\# \# p<0.01$, \#\#\#p <0.001 v. inactive lever; $n=18$ ). (B) Bilateral injection of the $\mathrm{CRF}_{1}$ receptor antagonist CP154526 into the CeA prevented cocaine-seeking behaviour induced by orexin-A administered to the pPVT (Sidak post hoc test; ${ }^{* * *} p<0.001 \mathrm{v}$. extinction and sham; $\# \# \#<0.001 \mathrm{v}$. inactive lever; $+++p<0.001 \mathrm{v}$. vehicle; $n=6-18)$. (C) Schematic illustration of injection site tracks for the pPVT (left) and CeA (right; $\mathrm{x}=$ rats with correct cannula placements; $\mathrm{O}=$ rats with incorrect cannula placements). $\mathrm{CeA}=$ central nucleus of the amygdala; $\mathrm{CP}=$ $\mathrm{CP} 154526$; $\mathrm{CRF}=$ corticotropin releasing factor; $\mathrm{pPVT}=$ posterior paraventricular nucleus of the thalamus; $\mathrm{SEM}=$ standard error of the mean .

The pPVT projects to the CeA, and neurons that express CRF have been found to have close contact with pPVT fibres ${ }^{31}$ Recent studies have confirmed the importance of PVT-to-CeA projections in adaptive responses to stress and anxiety. ${ }^{33-35}$ Our results showed that CeA activation occurred during seeking behaviour induced by OrxA administration to the pPVT only in C-LA rats, and not in the C-SA or sweetened condensed milk groups. Extended cocaine self-administration might induce changes in the CeA that are either quantitatively or qualitatively different from those induced by limited cocaine or sweetened condensed milk self-administration. Such differences could explain the differential recruitment of the $\mathrm{CeA}$ during seeking behaviour induced by OrxA administration in the C-LA, C-SA and sweetened condensed milk groups. Supporting this possibility, the CeA has been implicated in the regulation of negative affective states that are typically experienced during withdrawal or extensive drug use (for review, see $\left.\mathrm{Koob}^{36}\right)$. Therefore, extended cocaine exposure and subsequent withdrawal can induce long-lasting cellular and 
molecular alterations of CeA neurons, contributing to progressively worse negative affective states during withdrawal from cocaine. For example, the membrane excitability of CeA neurons exhibited transient adaptation following short exposure to cocaine, and these adaptations persisted following extended access to cocaine. ${ }^{37}$ Moreover, rats that were exposed to extended-access cocaine selfadministration exhibited long-lasting increases in $\alpha$-amino3-hydroxy-5-methyl-4-isoxazolepropionic acid and $\mathrm{N}$ methyl-D-aspartate glutamatergic receptor subunit expression in the $\mathrm{CeA}^{38}$ and decreased glutamate turnover ${ }^{39}$ whereas rats that exhibited compulsive increases in cocaine self-administration had higher $\gamma$-aminobutyric acid (GABA)-ergic tone in the $\mathrm{CeA},{ }^{40,41}$ consistent with earlier findings that neurons in the CeA exhibited persistent adaptations of membrane excitability following extended exposure to cocaine. ${ }^{37}$ Persistent cocaine-seeking observed following chronic cocaine exposure is dependent on CeA recruitment, ${ }^{42}$ which may contribute to the long-lasting negative affective states that are characteristic of cocaine withdrawal. Optogenetic stimulation of the $\mathrm{CeA}$ intensified the motivation for cocaine, whereas pharmacological or optogenetic inhibition of the CeA suppressed cocaine intake, ${ }^{43}$ decreased footshock-induced cocaine-seeking ${ }^{44}$ and decreased the incubation of cocaine craving in a self-administration procedure, ${ }^{45,46}$ suggesting that functionality of the $\mathrm{CeA}$ is critical for maintaining the motivation for cocaine.

The involvement of $\mathrm{CRF}_{1}$ receptors in the CeA during cocaine-seeking behaviour that was identified in the present study was consistent with an extensive body of literature that supports a functional role for $\mathrm{CRF}_{1}$ receptors in negative emotional states and the development of $\mathrm{CRF}_{1}$ receptor antagonists to treat affective-like disorders and substance use disorders. ${ }^{47-49}$ Systemic injections of $\mathrm{CRF}_{1}$ receptor antagonists reduced the escalation of alcohol intake in dependent rats and mice ${ }^{50,51}$ and attenuated increases in the selfadministration of cocaine, ${ }^{52}$ nicotine ${ }^{53}$ and heroin ${ }^{54}$ in rats with a history of extended access to the drugs. The majority of CeA neurons activated during alcohol withdrawal were shown to express CRF, and the optogenetic inactivation of CeA CRF neurons or blockade of $\mathrm{CRF}_{1}$ receptors in the CeA decreased alcohol intake in dependent rats. ${ }^{55-57}$ Stressors that elicit CRF release reinstated cocaine-seeking behaviour more effectively in rats that had been previously exposed to extended-access cocaine self-administration than in rats that were exposed to cocaine under short-access conditions, and $\mathrm{CRF}_{1}$ receptor antagonists more effectively reduced cocaine self-administration in rats that had extended access to cocaine compared to their short-access counterparts. ${ }^{52,58}$ Our results, together with earlier anatomic studies, ${ }^{31}$ support the possibility that intra-pPVT administration of OrxA influences the activity of $\mathrm{CeA}$ neurons through $\mathrm{CRF}_{1}$ to induce the reinstatement of cocaine-seeking behaviour in cocainedependent rats. Further supporting this hypothesis is an earlier study that showed that the intracerebroventricular administration of a nonselective CRF receptor antagonist attenuated anxiety-like behaviour that was produced by an injection of OrxA in the PVT..$^{59}$ Overall, our findings suggest that OrxA administration in the pPVT induced the reinstatement of cocaine-seeking behaviour in C-LA rats via $\mathrm{CRF}_{1}$ receptor signalling in the CeA. Although we observed no CeA activation in the C-SA and sweetened condensed milk groups, the involvement of this brain region and the implication of $\mathrm{CRF}_{1}$ during reinstatement induced by OrxA administration to the pPVT cannot be completely excluded. One possibility could be that the CeA played a key role in reinstatement in the C-SA and sweetened condensed milk groups that could not be detected simply with Fos labelling. As well, brain regions other than the CeA could also have been directly responsible for rewardseeking behaviour in the C-SA and sweetened condensed milk groups. One hypothesis was that OrxA injections in the PPVT may have induced reward-seeking behaviour in the C-SA and/or sweetened condensed milk groups via recruitment of the nucleus accumbens shell. Supporting this possibility are earlier findings that PVT neurons send projections to the nucleus accumbens shell ${ }^{2}$ and that orexin terminals make contact with PVT neurons that project to the nucleus accumbens shell. ${ }^{60}$ Moreover, the percentage of PVT neurons that were activated and projected to the nucleus accumbens shell increased after the context-induced renewal of alcohol-seeking. ${ }^{2}$ Although the primary focus of this work was to investigate the C-LA group, it will be important for the future to determine 2 things: which brain regions are specifically involved in seeking behaviour that is induced by intra-pPVT OrxA administration in C-SA and sweetened condensed milk groups; and whether $\mathrm{CRF}_{1}$ receptor signalling in the CeA plays a role in the reinstatement of reward-seeking behaviour in C-SA and sweetened condensed milk groups.

Although we observed an increase in Fos expression in the BNST in C-LA rats, the reason why recruitment of the BNST was not essential for cocaine-seeking behaviour induced by OrxA administered to the pPVT remains unclear. The CeA and BNST are interconnected brain structures. ${ }^{61}$ One could argue that the CeA and BNST control distinct functions with respect to stress and anxiety. The CeA and BNST play different roles in the regulation of anxiety and fear states, suggesting that the BNST mediates persistent anxiety-like responses, unlike the $\mathrm{CeA}$, which produces defensive behaviours in response to forthcoming threats. ${ }^{62}$ However, other studies have also shown that these 2 brain structures coordinate to encode aversive experiences in both acute and chronic responses to fear and anxiety. ${ }^{63-65}$ Nevertheless, although the BNST could play a role in promoting cocaine-seeking behaviour, activation of the BNST does not appear to be necessary to mediate cocaine-seeking behaviour induced by an OrxA injection in the pPVT. We also observed increases in Fos expression in the prelimbic cortex, infralimbic cortex and basolateral amygdala, not only following cocaine-seeking behaviour induced by OrxA administration to the pPVT but also when the rats were returned to their home cage. These observations suggest that the initial recruitment of these brain regions was attributable to direct activation following intra-pPVT OrxA injection, in 
contrast to our observations for the CeA and BNST. These findings further suggest that activation of the CeA and BNST during cocaine-seeking behaviour induced by intrapPVT OrxA administration could be indirect and will need to be investigated further.

The fact that we did not include female rats in the present study is a limitation. There are notable sex differences in cocaine addiction. Women differ from men on several measures of addictive behaviour, ranging from the temporal development of dependence to susceptibility to relapse. ${ }^{66}$ In rodents, female rats versus male rats acquire cocaine self-administration more quickly and exhibit greater resistance to extinction; ${ }^{67,68}$ they also show an increase in cocaine prime-induced reinstatement ${ }^{69}$ but similar cue- and stress-induced reinstatement. ${ }^{68,70,71}$ Evidence also shows sex differences in the orexin system, in which females exhibit higher activity than males. This contributes to sex differences in response to stress. ${ }^{72}$ However, only a few studies of the involvement of the orexin system in drug addiction have specifically examined sex differences. The orexin receptor 1 antagonist SB334867 attenuated cocaine cue-induced reinstatement and cocaine drug + cue prime-induced reinstatement in males, but orexin receptor 1 blockade had no effect in females. ${ }^{73}$ Males were sensitive to the effects of moderate doses of SB334867 on stress-induced reinstatement and the combination of stress + cue, whereas female rats exhibited a decrease in reinstatement only at higher SB334867 doses. ${ }^{73}$ Although the exact mechanisms that underlie these sex differences are unclear, gonadal hormones may play a critical role. Estrogen facilitates drug-seeking and locomotor sensitization, and progesterone counteracts the effects of estrogen. ${ }^{74}$ Sex and estrous-cycle differences in the orexin system have been reported. Female rats express higher levels of OrxA and orexin receptor 1 in the hypothalamus compared to male rats, ${ }^{75,76}$ and the hypothalamic expression of orexin receptors increases selectively in female rats during proestrus compared to other estrous cycle stages. ${ }^{77}$ Males and females could exhibit similar reinstatement of cocaine-seeking or sweetened condensed milk-seeking; but assuming that an increase in orexin receptor expression is similar between the PVT and hypothalamus, depending on the estrous cycle, females could be more sensitive to the effect of pPVT OrxA prime-induced reinstatement (i.e., the same dose of OrxA could induce a stronger effect than what we observed for males) and exhibit stronger activation of both the CeA and BNST. A thorough examination of specific brain regions that are recruited by intra-pPVT OrxA administration in female rats and the importance of their functionality during cocaineseeking behaviour are necessary to better understand the neurobiology of cocaine-seeking behaviour induced by intra-pPVT OrxA administration.

\section{Limitations}

The potential limitations of this study included the following: the use of male rats only; the use of an indirect approach to test the hypothesis that OrxA administered to the pPVT drives cocaine-seeking via $\mathrm{CRF}_{1}$ receptor signalling in the $\mathrm{CeA}$; and the lack of analysis of the participation of CeA subregions.

\section{Conclusion}

The results of the present study showed that cocaine-seeking behaviour induced by OrxA administered to the pPVT was driven by the activation of $\mathrm{CeA} \mathrm{CRF}_{1}$ receptors in rats that were previously exposed to extended-access cocaine. These data provide novel insights into the mechanism that underlies cocaine craving and relapse, and the participation of the hypothalamus-PVT-CeA axis.

Acknowledgements: This is publication number 29991 from The Scripps Research Institute. The authors thank Michael Arends for assistance with manuscript preparation. This study was supported by the National Institute on Drug Abuse and National Institute on Alcohol Abuse and Alcoholism (grant no. DA033344, AA024146, AA006420, and AA026999 to RM-F).

Affiliations: From The Scripps Research Institute, La Jolla, California, USA (Matzeu, Martin-Fardon).

Competing interests: None declared.

Contributors: A. Matzeu and R. Martin-Fardon designed the study. A. Matzeu acquired the data, which A. Matzeu and R. Martin-Fardon analyzed. A. Matzeu and R. Martin-Fardon wrote the article, which both authors reviewed. Both authors approved the final version to be published and can certify that no other individuals not listed as authors have made substantial contributions to the paper.

Content licence: This is an Open Access article distributed in accordance with the terms of the Creative Commons Attribution (CC BY-NC-ND 4.0) licence, which permits use, distribution and reproduction in any medium, provided that the original publication is properly cited, the use is noncommercial (i.e., research or educational use), and no modifications or adaptations are made. See: https://creativecommons.org/licenses/by-nc-nd/4.0/

\section{References}

1. Kirouac GJ. Placing the paraventricular nucleus of the thalamus within the brain circuits that control behavior. Neurosci Biobehav Rev 2015;56:315-29.

2. Hamlin AS, Clemens KJ, Choi EA et al. Paraventricular thalamus mediates context-induced reinstatement (renewal) of extinguished reward seeking. Eur J Neurosci 2009;29:802-12.

3. James MH, Charnley JL, Flynn JR et al. Propensity to "relapse" following exposure to cocaine cues is associated with the recruitment of specific thalamic and epithalamic nuclei. Neuroscience 2011; 199:235-42.

4. Bubser M, Deutch AY. Stress induces Fos expression in neurons of the thalamic paraventricular nucleus that innervate limbic forebrain sites. Synapse 1999;32:13-22.

5. Matzeu A, Cauvi G, Kerr TM et al. The paraventricular nucleus of the thalamus is differentially recruited by stimuli conditioned to the availability of cocaine versus palatable food. Addict Biol 2017;22:70-7.

6. Matzeu A, Weiss F, Martin-Fardon R. Transient inactivation of the posterior paraventricular nucleus of the thalamus blocks cocaineseeking behavior. Neurosci Lett 2015;608:34-9.

7. Kirouac GJ, Parsons MP, Li S. Orexin (hypocretin) innervation of the paraventricular nucleus of the thalamus. Brain Res 2005 . 1059:179-88.

8. Kirouac GJ, Parsons MP, Li S. Innervation of the paraventricular nucleus of the thalamus from cocaine- and amphetamineregulated transcript (CART) containing neurons of the hypothalamus. J Comp Neurol 2006;497:155-65. 
9. Peyron C, Tighe DK, Van Den pol AN et al. Neurons containing hypocretin (orexin) project to multiple neuronal systems. J Neurosci 1998;18:9996-10015.

10. Sutcliffe JG, de Lecea L. The hypocretins: excitatory neuromodulatory peptides for multiple homeostatic systems, including sleep and feeding. J Neurosci Res 2000;62:161-8.

11. Mieda M, Yanagisawa M. Sleep, feeding, and neuropeptides: roles of orexins and orexin receptors. Curr Opin Neurobiol 2002; 12:339-45.

12. Berridge CW, Espana RA, Vittoz NM. Hypocretin/orexin in arousal and stress. Brain Res 2010;1314:91-102.

13. Harris GC, Wimmer M, Aston-Jones G. A role for lateral hypothalamic orexin neurons in reward seeking. Nature 2005;437:556-9.

14. Martin-Fardon R, Zorrilla EP, Ciccocioppo R et al. Role of innate and drug-induced dysregulation of brain stress and arousal systems in addiction: focus on corticotropin-releasing factor, nociceptin/orphanin FQ, and orexin/hypocretin. Brain Res 2010;1314:145-61.

15. Martin-Fardon R, Cauvi G, Kerr TM et al. Differential role of hypothalamic orexin/hypocretin neurons in reward seeking motivated by cocaine versus palatable food. Addict Biol 2018;23:6-15.

16. Ishibashi $\mathrm{M}$, Takano S, Yanagida $\mathrm{H}$ et al. Effects of orexins/ hypocretins on neuronal activity in the paraventricular nucleus of the thalamus in rats in vitro. Peptides 2005;26:471-81.

17. Matzeu A, Kerr TM, Weiss F et al. Orexin-A/hypocretin-1 mediates cocaine-seeking behavior in the posterior paraventricular nucleus of the thalamus via orexin/hypocretin receptor-2.J Pharmacol Exp Ther 2016;359:273-9.

18. Matzeu A, Kallupi M, George O et al. Dynorphin counteracts orexin in the paraventricular nucleus of the thalamus: cellular and behavioral evidence. Neuropsychopharmacology 2018;43:1010-20.

19. Hsu DT, Kirouac GJ, Zubieta JK et al. Contributions of the paraventricular thalamic nucleus in the regulation of stress, motivation, and mood. Front Behav Neurosci 2014;8:73.

20. Heilig M, Koob GF. A key role for corticotropin-releasing factor in alcohol dependence. Trends Neurosci 2007;30:399-406.

21. Koob GF, Zorrilla EP. Neurobiological mechanisms of addiction: focus on corticotropin-releasing factor. Curr Opin Investig Drugs 2010;11:63-71.

22. National Research Council (US) Institute for Laboratory Animal Research. Guide for the care and use of laboratory animals. Washington (DC); National Academies Press; 1996.

23. Matzeu A, Martin-Fardon R. Blockade of orexin receptors in the posterior paraventricular nucleus of the thalamus prevents stressinduced reinstatement of reward-seeking behavior in rats with a history of ethanol dependence. Front Integr Nuerosci 2020; 14:599710.

24. James MH, Charnley JL, Levi EM et al. Orexin-1 receptor signalling within the ventral tegmental area, but not the paraventricular thalamus, is critical to regulating cue-induced reinstatement of cocaine-seeking. Int J Neuropsychopharmacol 2011;14:684-90.

25. Dayas CV, McGranahan TM, Martin-Fardon R et al. Stimuli linked to ethanol availability activate hypothalamic CART and orexin neurons in a reinstatement model of relapse. Biol Psychiatry 2008; 63:152-7.

26. Heydendael W, Sharma K, Iyer V et al. Orexins/hypocretins act in the posterior paraventricular thalamic nucleus during repeated stress to regulate facilitation to novel stress. Endocrinology 2011; $152: 4738-52$

27. Zhou K, Zhu Y. The paraventricular thalamic nucleus: a key hub of neural circuits underlying drug addiction. Pharmacol Res 2019; 142:70-6.

28. Yeoh JW, James MH, Jobling P et al. Cocaine potentiates excitatory drive in the perifornical/lateral hypothalamus. J Physiol 2012; 590:3677-89.

29. Yeoh JW, James MH, Graham BA et al. Electrophysiological characteristics of paraventricular thalamic (PVT) neurons in response to cocaine and cocaine- and amphetamine-regulated transcript (CART). Front Behav Neurosci 2014;8:280.

30. Moga MM, Weis RP, Moore RY. Efferent projections of the paraventricular thalamic nucleus in the rat. J Comp Neurol 1995; 359:221-38.

31. Li S, Kirouac GJ. Projections from the paraventricular nucleus of the thalamus to the forebrain, with special emphasis on the extended amygdala. J Comp Neurol 2008;506:263-87.
32. Vertes RP, Hoover WB. Projections of the paraventricular and paratenial nuclei of the dorsal midline thalamus in the rat. J Comp Neurol 2008;508:212-37.

33. Do-Monte FH, Quinones-Laracuente K, Quirk GJ. A temporal shift in the circuits mediating retrieval of fear memory. Nature 2015;519:460-3.

34. Penzo MA, Robert V, Tucciarone J et al. The paraventricular thalamus controls a central amygdala fear circuit. Nature 2015;519:455-9.

35. Ehrlich I, Humeau Y, Francois G et al. Amygdala inhibitory circuits and the control of fear memory. Neuron 2009;62:757-71.

36. Koob GF. Brain stress systems in the amygdala and addiction. Brain Res 2009:1293:61-75.

37. Chen B, Ma YY, Wang Y et al. Cocaine-induced membrane adaptation in the central nucleus of amygdala. Neuropsychopharmacology 2013;38:2240-8.

38. Lu L, Dempsey J, Shaham Y et al. Differential long-term neuroadaptations of glutamate receptors in the basolateral and central amygdala after withdrawal from cocaine self-administration in rats. J Neurochem 2005;94:161-8.

39. Roura-Martinez D, Ucha M, Orihuel J et al. Central nucleus of the amygdala as a common substrate of the incubation of drug and natural reinforcer seeking. Addict Biol 2020;25:e12706.

40. Kallupi M, Wee S, Edwards S et al. Kappa opioid receptormediated dysregulation of gamma-aminobutyric acidergic transmission in the central amygdala in cocaine addiction. Biol Psychiatry 2013;74:520-8.

41. Schmeichel BE, Herman MA, Roberto M et al. Hypocretin neurotransmission within the central amygdala mediates escalated cocaine self-administration and stress-induced reinstatement in rats. Biol Psychiatry 2017:81:606-15.

42. Murray JE, Beli-Rauscent A, Simon M et al. Basolateral and central amygdala differentially recruit and maintain dorsolateral striatumdependent cocaine-seeking habits. Nat Commun 2015;6:10088.

43. Warlow SM, Robinson MJF, Berridge KC. Optogenetic central amygdala stimulation intensifies and narrows motivation for Cocaine. J Neurosci 2017;37:8330-48.

44. McFarland K, Davidge SB, Lapish CC et al. Limbic and motor circuitry underlying footshock-induced reinstatement of cocaineseeking behavior. J Neurosci 2004;24:1551-60.

45. Lu L, Hope BT, Dempsey J et al. Central amygdala ERK signaling pathway is critical to incubation of cocaine craving. Nat Neurosci 2005;8:212-9.

46. Lu L, Uejima JL, Gray SM et al. Systemic and central amygdala injections of the mGluR(2/3) agonist LY379268 attenuate the expression of incubation of cocaine craving. Biol Psychiatry 2007; 61:591-8.

47. Zorrilla EP, Koob GF. The therapeutic potential of CRF1 antagonists for anxiety. Expert Opin Investig Drugs 2004;13:799-828.

48. Zorrilla EP, Koob GF. Progress in corticotropin-releasing factor-1 antagonist development. Drug Discov Today 2010;15:371-83.

49. Zorrilla EP, Heilig M, De Wit $\mathrm{H}$ et al. Behavioral, biological, and chemical perspectives on targeting $\mathrm{CRF}(1)$ receptor antagonists to treat alcoholism. Drug Alcohol Depend 2013;128:175-86.

50. Chu K, Koob GF, Cole M et al. Dependence-induced increases in ethanol self-administration in mice are blocked by the CRF1 receptor antagonist antalarmin and by CRF1 receptor knockout. Pharmacol Biochem Behav 2007;86:813-21.

51. Gilpin NW, Richardson HN, Koob GF. Effects of CRF1-receptor and opioid-receptor antagonists on dependence-induced increases in alcohol drinking by alcohol-preferring $(\mathrm{P})$ rats. Alcohol Clin Exp Res 2008;32:1535-42.

52. Specio SE, Wee S, O'Dell LE et al. CRF(1) receptor antagonists attenuate escalated cocaine self-administration in rats. Psychopharmacology (Berl) 2008;196:473-82.

53. George O, Ghozland S, Azar MR et al. CRF-CRF1 system activation mediates withdrawal-induced increases in nicotine selfadministration in nicotine-dependent rats. Proc Natl Acad Sci U S A 2007;104:17198-203.

54. Greenwell TN, Funk CK, Cottone P et al. Corticotropin-releasing factor-1 receptor antagonists decrease heroin self-administration in long- but not short-access rats. Addict Biol 2009;14:130-43.

55. Varodayan FP, De Guglielmo G, Logrip ML et al. Alcohol dependence disrupts amygdalar L-type voltage-gated calcium channel mechanisms. J Neurosci 2017;37:4593-603.

56. Funk CK, O'Dell LE, Crawford EF et al. Corticotropin-releasing factor within the central nucleus of the amygdala mediates enhanced 
ethanol self-administration in withdrawn, ethanol-dependent rats. J Neurosci 2006;26:11324-32

57. de Guglielmo G, Kallupi M, Pomrenze MB et al. Inactivation of a CRF-dependent amygdalofugal pathway reverses addiction-like behaviors in alcohol-dependent rats. Nat Commun 2019;10:1238.

58. Mantsch JR, Baker DA, Francis DM et al. Stressor- and corticotropin releasing factor-induced reinstatement and active stress-related behavioral responses are augmented following long-access cocaine self-administration by rats. Psychopharmacology (Berl) 2008; 195:591-603

59. $\mathrm{Li} \mathrm{Y}, \mathrm{Li} \mathrm{S}, \mathrm{Wei} \mathrm{C}$ et al. Orexins in the paraventricular nucleus of the thalamus mediate anxiety-like responses in rats. Psychopharmacology (Berl) 2010;212:251-65.

60. Parsons MP, Li S, Kirouac GJ. The paraventricular nucleus of the thalamus as an interface between the orexin and CART peptides and the shell of the nucleus accumbens. Synapse 2006;59:480-90.

61. de Olmos JS, Heimer L. The concepts of the ventral striatopallidal system and extended amygdala. Ann N Y Acad Sci 1999; 877:1-32.

62. Walker DL, Miles LA, Davis M. Selective participation of the bed nucleus of the stria terminalis and CRF in sustained anxiety-like versus phasic fear-like responses. Prog Neuropsychopharmacol Biol Psychiatry 2009;33:1291-308.

63. Fox AS, Kalin NH. A translational neuroscience approach to understanding the development of social anxiety disorder and its pathophysiology. Am J Psychiatry 2014;171:1162-73.

64. Fox AS, Oler JA, Tromp DPM et al. Extending the amygdala in theories of threat processing. Trends Neurosci 2015;38:319-29.

65. Gungor NZ, Pare D. Functional heterogeneity in the bed nucleus of the stria terminalis. J Neurosci 2016;36:8038-49.

66. Fattore L, Altea S, Fratta W. Sex differences in drug addiction: a review of animal and human studies. Womens Health (Lond Engl) 2008;4:51-65.
67. Lynch WJ, Carroll ME. Sex differences in the acquisition of intravenously self-administered cocaine and heroin in rats. Psychopharmacology (Berl) 1999;144:77-82.

68. Fuchs RA, Evans KA, Mehta RH et al. Influence of sex and estrous cyclicity on conditioned cue-induced reinstatement of cocaine-seeking behavior in rats. Psychopharmacology (Berl) 2005;179:662-72.

69. Kippin TE, Fuchs RA, Mehta RH et al. Potentiation of cocaineprimed reinstatement of drug seeking in female rats during estrus. Psychopharmacology (Berl) 2005;182:245-52

70. Feltenstein MW, Henderson AR, See RE. Enhancement of cueinduced reinstatement of cocaine-seeking in rats by yohimbine: sex differences and the role of the estrous cycle. Psychopharmacology (Berl) 2011;216:53-62.

71. Becker JB, Koob GF. Sex differences in animal models: focus on addiction. Pharmacol Rev 2016;68:242-63.

72. Grafe LA, Bhatnagar S. The contribution of orexins to sex differences in the stress response. Brain Res 2020;1731:145893

73. Zhou L, Ghee SM, Chan C et al. Orexin-1 receptor mediation of cocaine seeking in male and female rats. J Pharmacol Exp Ther 2012; 340:801-9.

74. Quinones-Jenab V, Jenab S. Progesterone attenuates cocaine-induced responses. Horm Behav 2010;58:22-32.

75. Taheri S, Mahmoodi M, Opacka-Juffry J et al. Distribution and quantification of immunoreactive orexin $\mathrm{A}$ in rat tissues. FEBS Lett 1999;457:157-61.

76. Johren O, Neidert SJ, Kummer $\mathrm{M}$ et al. Prepro-orexin and orexin receptor mRNAs are differentially expressed in peripheral tissues of male and female rats. Endocrinology 2001;142:3324-31.

77. Silveyra P, Cataldi NI, Lux-Lantos V et al. Gonadal steroids modulated hypocretin/orexin type-1 receptor expression in a brain region, sex and daytime specific manner. Regul Pept 2009; 158:121-6. 